\title{
PROOF-NETS AND DEPENDENCIES
}

\author{
Alain LECOMTE \\ GRII \\ Université Blaise Pascal \\ 34 Avenue Carnot \\ 63037- CLERMONT-FERRAND cedex \\ (France)
}

\begin{abstract}
Proof-Nets (Roorda 1990) are a good device for processing with categorial grammars, mainly because they avoid spurious ambiguities. Nevertheless, they do not provide easily readuble structures and they hide the true proximity between Categorial Grammars and Dependency Grammars. We give here an other kind of Proof-Nets which is much related to Dependency Structures similar to those we rneet in, for instance (Hudson 1984). These new Proof-Nets are called Connection Nets. We show that Connection Nets provide not only easily interpretable structures, but also that processing with them is more efficient. 1
\end{abstract}

\section{Introduction}

Nowadays, two formalisms are very attractive in Natural Language Processing:

$$
\text { - Categorial Grammars, and }
$$$$
\text { - Dependency Grammars. }
$$

Numerous studies try to shed light on their similarities and differences. We may quote for instance works by Hudson (1984, 1990), Barry \& Pickering(1990), Hausser (1990). Hepple (1991). One interesting particularity common to these two formalisms seems to be the capacity of leading to an incremental processing, which, in tur, leads to an on-line processing.

Moreover, these formalisms are now very well known. Categorial Grammars have been much studied recently, particularly since the article of Ades and Steedman (1982) and the re-discovering of previous works done by Lambek $(1958,1961)$. The most comprehensive form taken by Categorial Grammars is the Lambck Calculus, studied by many authors like Moortgat (1988, 1990), Buszkowski (1986, 1988), Desclés (1990) ctc. Since the recent work by J-Y Girard (see for instance Girard 1987), which led to the framework of Linear Logic, it has become apparent that the Lambek Calculus amounts to a non-commutative version of a sub-system of Linear Logic, where a structural rule forbids sequents with an empty antecedent.

Semantic properties of this system have been studied by Buszkowski (1986, 1988) and Wansing (1990). Two models are often given: onc consists of residuation semigroups spread over free semigroups, and another one is given by the directional typed lambda-calculus.

1 I em indebted to Dirk Roorda for fruitful discussions during a brief visit I made in Amsterdam in Spring 1991
Dependency Grammars are originating from earlier works by the French linguist Tesniere (1965). They were theoretically studied by Gaifman, who demonstrated theorems on the Generative Capacity of Dependency Grammars. We will consider here that the formalism of "Word Grammar" (Hudson 1984, 1990) is representative of this trend.

Our purpose in this communication is to show that building dependency structures gives an other kind of semantics for the Lambek Calculus and various subsystems. This semantics is useful in that it will allow us to conceive extensions of the Lambek Calculus. Moreover, the correspondance proposed between these two aspects provides us with a method of parsing related to the conception of "parsing as deduction", together with a method for avoiding spurious ambiguities. We will show that it is isomorphic to the method of proof-nets (Girard 1987, Danos and Regnier 1989, Roorda 1990, 1991), but that it has the advantage over this last method of being more efficient and of providing more clarity on the result of processing. The devices we obtain are more readable, because they are interpretable in terms of dependency structures. Otherwise, the parsing method can be an incremental one.

\section{The Method of Proof-Nets in the Lambek Calculus}

The problem of spurious ambiguities in Categorial Grammar is very often discussed (see for instance Hendriks and Roorda (1991)). A proof-net is a device which contains all the equivalent proofs of the same result. As Roorda (1990) says: "A proof-net can be viewed as a parallellized sequent proof [...] It is a concrete structure, not merely an abstract equivalence class of derivations, and surely not a special derivation with certain constraints on the order in which the rules must be applied."

The principles of construction of proof-ncts are related to the inference rules of the Lambek Calculus, when it is viewed as a sequent calculus. If we here omit the product, we have the following rules, which belong to two different lypes: 
Binary rules (or type-2 rules):

(where $\boldsymbol{\theta}$ is a non-empty sequence of categories, and $\Gamma$ and $\Lambda$ are arbitrary sequences of categories) ${ }^{2}$

[L/]:

$$
\begin{gathered}
\Theta \rightarrow B \Gamma, A, \Lambda \rightarrow C \\
\Gamma, A / B, \theta, \Lambda \rightarrow C
\end{gathered}
$$

$[\mathrm{LX}]:$

$$
\frac{\theta \rightarrow B \Gamma, A, \wedge \rightarrow C}{\Gamma, \theta, B \backslash A, \wedge \rightarrow C}
$$

Unary rules (or type- 1 rules): ( $\Gamma$ is non empty)

$$
[R /]: \quad \begin{array}{ll}
\Theta, B \rightarrow A \\
\hdashline \rightarrow A / B
\end{array} \quad[R]: \quad \frac{B, \Theta \rightarrow A}{\theta \rightarrow B M A}
$$

In these nules, contexts $(\theta, \Gamma, \Lambda)$ are "static". That means that they can neither be contracted, nor expanded, nor permuted. They play no role in the application of rules. So, it is convenient to "forget" them and to represent the rules according to schemes similar to:

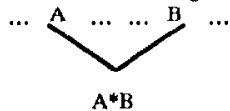

Such a scheme is called $a$ link. Other types of links are provided by the identity axiom: [ax] $\mathrm{A} \rightarrow \mathrm{A}$, which becomes:

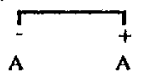

and by the cut-rule:

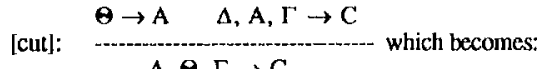

$$
\begin{aligned}
& \Delta, \boldsymbol{\theta}, \Gamma \rightarrow \mathrm{C} \\
& + \\
& \text { A } \quad \text { A }
\end{aligned}
$$

Links asscxiated to rules belong to two types:

type-2 links corresponding to type-2 rules (depicted by lines)

type-1 links corresponding to type-1 rules (depicted by dashed lines)

D. Roorda (1990) has shown the following theorem:

Theorem: (Soundness and Completeness of Proof-Nets w.r.t. L.)

2 We use here the notation introduced by J. Lambek, according to which the argument category is always under the slash, in such a way that $A / B$ means a category which becomes an $A$ if a $B$ is met on the right, and $B \backslash A$ a category which becomes an $A$ if a $B$ is met on the left.
If the rules $[\mathrm{L}],[\mathrm{L} /],[\mathrm{R} \backslash],[\mathrm{R} /]$ are represented by the following links:

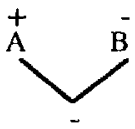

A $\backslash B$<smiles>[3H][I-][Te]</smiles>

AVB<smiles>[3H]CC[3H]</smiles>

$\mathrm{B} / \mathrm{A}$ then a sequent $\Gamma \rightarrow \Delta$ is a theorem of the (Product free) Lambek Calculus if and only if we can build, starting with this sequent and applying links recursively, a connected planar graph having the following property: for each application of a type- 1 link, every suppression of one of the two dashed lines leaves the graph connected.

Examples:

$a \rightarrow b /(a \backslash b)$ is a theorem: sec figure (1) below.

$b /(a \backslash b) \rightarrow$ a is not a theorem: see figure (2) below.

figure (1):

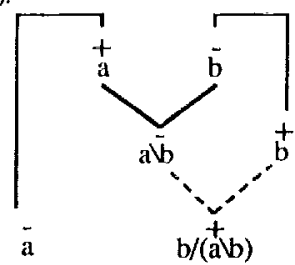

figure (2):

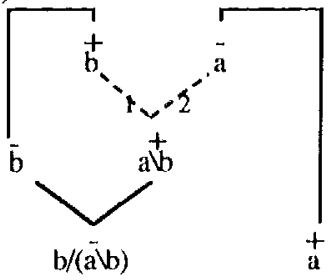

In this last case, we see that the suppression of the edge 2 leads to disconnection.

\section{Dependency Structures}

A dependency structure associated to a sentence is a tree on the words of this sentence. Edges represent dependency links such that the source of an edge is considered as the head and the target as a dependant. Hudson (1984) gives criteria to distinguish heads and dependants. It is an open question whether a head can be viewed as a functor, the dependant being viewed as an argument. The facts that criteria involve agreement and 
that according to the Keenan's thesis: "functors agree with their argument" seem in favour of identification. But other scholars disagree, like Moortgat and Morrill, who introduce in their recent works, four notions: head, dependant, functor and argument. Nevertheless, we will accept the first thesis in the following, adopting the conception of Barry and Pickering (1990) on this subject.

Another problem appears in the necessity of accounting for structures with multiplicity of heads (in the case of the control of infinitives for instance) because this necessity leads to graphs which are no longer trees, but dags.

We assume that a dependency structure is a graph on words. In a first step, we will consider only trees. The approach will be that of a semantic interpretation in terms of trees, similar to what we do when we give a semantic interpretation of logic formulae in terms of sets. The usual operators like / and $\backslash$ will be interpreted as connection operations in an algebra of trees. In a second step, we will have to modify this interpretation in order to obtain not only application and composition but division too.

\section{Operations on Trees}

We start with a set of directed trees associated to lexical entries. (see figure (3) below).

figure (3)

connait:

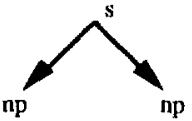

promet

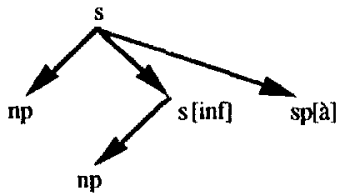

These trees are called initial trees. The initial state of a representation of the structure of a sentence consists in an ordered sequence of these initial trees. Then, at each step. we build a new tree obtained by connection of previous trees. These operations ane: (cf Lecomte 1990)

$$
\text { - lefi-linkage }
$$$$
\text { - right-linkage }
$$

A tree $\mathrm{G}_{1}$ is right (resp. lefi) linkable to a tree $\mathrm{G}_{2}$ iff: 1) $\mathrm{G}_{1}$ and $\mathrm{G}_{2}$ are adjacent, $\mathrm{G}_{2}$ being adjacent to the right (resp, left) of $\mathrm{G}_{1}$

2) $\mathrm{G}_{\text {I }}$ has a rightmost (resp leftmost) branch the first edge of which is right (resp left) directed and the maximal sub-tree attached to this first edge entirely covers a continuous subtree of $\mathrm{G}_{2}$.

The by-product of the right-linkage (resp left-linkage) of $G_{1}$ with $G_{2}$, when $G_{1}$ is right (resp left) linkable to $G_{2}$ is the tree $G_{3}$ obtained as the union of $G_{1}$ and $G_{2}$, modified in the following way:
The rightmost right-directed (resp. leftmost left-directed) first-level edge of $G_{1}$ is connected to the root of $G_{2}$, and the subtree of $\mathrm{G}_{2}$ covered by the maximal subtree attached to this edge is said to be marked in $\mathrm{G}_{2}$. Left (resp. right)-directed edges of $\mathrm{G}_{2}$ which are not marked remain free and take precedence in the left-to-right (resp right-to-left) order of first-level edges over those remaining free in $\mathrm{G}_{1}$.

We can introduce restrictions on these operations:

we will call restriction-AB the following constraint: the subtree of $\mathrm{G}_{2}$ covered by the subtree of $\mathrm{G}_{1}$ must be identical to the whole tree $\mathrm{G}_{2}$,

restriction-C: at most the rightmost (resp leftmost) branch of $\mathrm{G}_{2}$ may be uncovered.

restriction-Crec: a right (resp left) subtree of $\mathrm{G}_{2}$ may be uncovered

restriction-Cmix: at most the rightmost (resp leftmost) or the leftmost (resp rightmost) branch of $\mathrm{G}_{2}$ may bc uncovered

Definition: we call connection tree every initial tree and every tree obtained by the application of linkage operations on earlier connection trees (according to eventual restrictions).

We claim that such a system gives an interpretation of very simple categorial grammars, depending on the restrictions we select. Like similar constructions (Steedman 1991) where general principles such as Adjacency, Directional Consistency and Directional Inheritance are explained in terms of a more detailed analysis of categories, this system is suited to express such generalities. Because of the structure of linkage operations, these principles are obvious, Adjacency and Directional Consistency are contained in the definition. Directional Inheritance comes from the fact that we never allow to change anything in the labels of edges (the fact that they are left or right directed). We only allow to change the status of an edge (free to bound or marked). In so doing, we reach, like Steedman does, the conclusion that so-called Dysharmonic Composition Rules are consistent with these principles (even if they are not with the Lambek Calculus!).

A connection system eliminates spurious ambiguities because when they are bound, links are undefeasable : there is no way of re-doing something that was primilarly done with success. In this respect, the calculus on trees concurs with the well known method of chart-parsing. (see figure (4): there is only one tree for two reductions by means of Cancellation Schemes). 
figure (4):

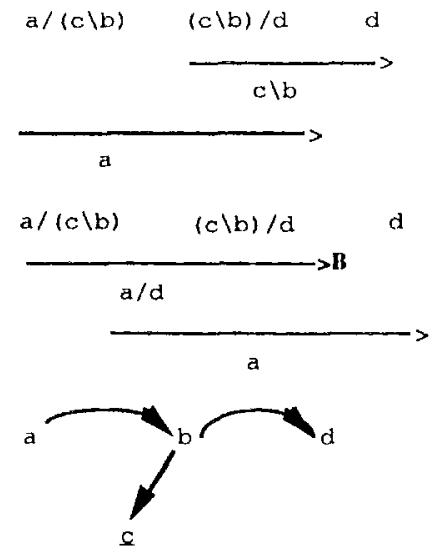

Moreover, a connection system provides us with a semantics for Dependency-Constituency Grammars, in the tradition of Barry and Pickering (1990) and Mark Hepple (1991).

\section{Connection and Identification: an Extension of Connection Systems}

\section{J.The Need for Division Rules}

It is obvious that the previous system does not include any kind of Division Rules or any kind of Type-Raising Rule. So, it cannot provide any analysis for sentences with extraction, as for instance:

\section{le livre dont je connais le titre est sur la table}

(the book the title of which I know is on the table)

because in such an analysis, we have to transform a regular $n$ (titre) into a functorial category which requires a noun-modifier on its right (n/(nv)) .

We shall define a new connection system which is a conservative extension of the previous one (except for the admissibility of Dysharmonic Rules). We will call it: the Connection Net System.

As for the proof-nets, we want to demonstrate theorems that have a sequent form like: $\Gamma \rightarrow X$, where $\Gamma$ is a non empty sequence of categories and $X$ is a category. Wc distinguish two kinds of connection trees: those which are on the right-hand side of the scquent we want to demonstrate, and those which are on the left-hand side. When we are viewing the problems in a naturaldeduction way, we can say that the first are the trees $t o$ build and the second are those which are used in this task. We will call the firstright-trees and the second lefttrees. The set of left-trees and right-trees at any stage will be called a Construction Net.
Schematically, operations are not merely connections because connections can only expand elementary trees towards more complex ones. And we need operations to reduce the complexity of a tree. For instance, to show the usual rule of Type-Raising: $a \rightarrow b /(a b)$ we have to show that the right-tree associated to $b /(a b)$ reduces to something isomorphic to a. The fact that, generally, the converse $(b /(a b) \rightarrow a)$ is not true results from the fact that the same reduction is not possible when the same tree is put on the left-hand side. This exemplifies the fundamental dissymetry of the calculus.

\subsection{Type-1 Edges and Type-2 Edges}

We will then distinguish two sorts of edges and two sorts of nodes in a connection tree: type- 1 edges and nodes and type-2 edges and nodes.

Definition: A type-2 edge in a connection tree is:

- an odd level edge in a left-trec, or

- an even level edge in a right-tree

A type-1 edge in a connection tree is:

- an even level coge in a left-tree, or

- an odd level edge in a right-tree

A type-i $(\mathrm{i}=1,2)$ node is the target of a type-i exge.

Roots are type-1 nodes if in a left-trec, and type2 nodes if in a right-tree.

Two nodes are said to be complementary if they have not the same type.

Examples: figure (5)

a) a new tree assigned to a lexical entry:

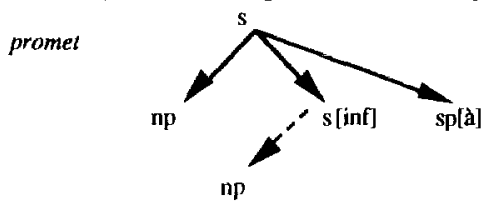

b) a pair $(L, R)$ associated to a sequent:

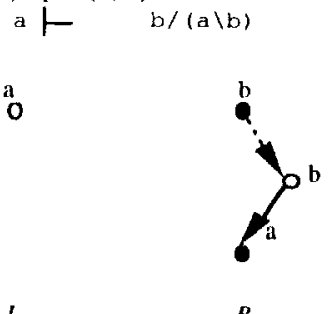

L $R$

Definition: we call identification link either a nondirected edge which links two identical nodes which are complementary, one in a left-trec, the other in a righttree, or a type-1 directed edge linking two complementary nodes having same label.

We call connection link every link we shall be able to establish, according to the following conventions, between a type-1 node, which is the ending point of a 
type-2 edge, and a type-2 node which does not belong to the same tree.

\subsection{Nodes-numbering}

Rule: each node of the initial construction net receives a number, called its degree, according to the following rules:

\section{- for a type-2 edge:}

if it is right directed, the degree of its source is less than the degree of all the nodes below it,

if it is left directed, the degree of its source is greater than the degree of all the nodes below it,

for two type-2 edges, children's degrees of the leftmost branch are less than those of the rightmost branch.

- for a type-1 edge:

if it is right directed, the degree of its source is greater than the degree of all the nodes below it,

if it is left directed, the degree of its source is less than the degree of all the nodes below it

for two type-1 edges, children's degrees of the rightmost branch are less than those of the leftmost branch.

The lowest degree of the right successor of an initial tree is the successor of the greatest degree of this latter tree.

Example of such a numbering: figure (6)

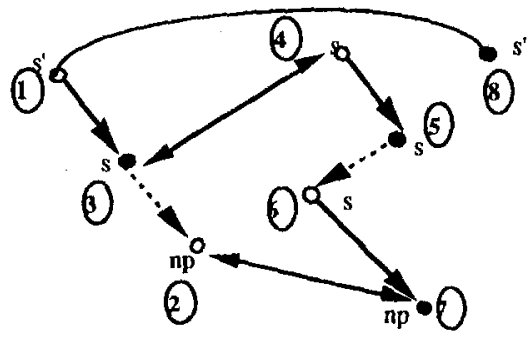

$L \quad R$

intervals.

$$
\begin{aligned}
& \text { s'-s' : }\left[11_{-} 8\right] \\
& \text { s-s : }\left[33_{-} 4\right] \\
& \text { np-np : }\left[2 \_7\right] \\
& \text { s-s : [5_6] }
\end{aligned}
$$

Each link is now associated to a pair of degrees, called its interval.

From now on, $L$ and $R$ will denote respectively: the left hand side and the right hand side of a Construction Net. The Construction Net will be denoted by: $\langle L \mid R\rangle$.

\subsection{Linking the Nodes}

Nodes will be linked according to the following principles:

COMPLEMENTARITY: two nodes are linked only if they have the same label and they are of complementary types.

NON-OVERLAP: the linking of all the nodes in the Construction Net must meet the non-overlap convention, which stipulates that given two arbitrary intervals, either one contains the other or they are disjoint.

Theorem: (Conservativity of Connection Operations) The Non-Overlap condition is a conservative extension of the conditions on connection (restriction $\mathrm{C}^{\mathrm{rec}}$ ) stipulated in 84. That means: every connection system based on $\mathrm{Crec}^{\mathrm{re}}$, when translated in the Connection Net System, follows this convention.

\section{Building a Correa $\mathrm{Ne}$}

Definition: Given an ordered sequence of left-trees L and a right-tree $R$, we will say that $L$ and $R$ yield $a$ correct net iff there is a linkage of all the nodes in the Construction Net $\langle\mathrm{L} \mid \mathrm{R}\rangle$, which gives a connected graph, respects the complementarity principle and the non-overlap principle, and is such that: when all the type-I edges are removed, the graph remains connected.

The fundamental result is the following:

Theorem: (Soundness and Completeness w.r.t. $\mathbf{A}^{3}$ ) Let $\Gamma \rightarrow A$ be a sequent expressed in the Product-Free Lambek Calculus, where $\Gamma$ is a non empty sequence of categories and $A$ is $a$ catcgory, let $L$ be the sequence of left connection trees associated to the elements of $\Gamma$ and $R$ be the right tree associated to $A$, the sequent is a theorem if and only if $L$ and $R$ yield a correct net. In other terms: the Connection Net System is sound and complete w.r.t. the Product-Free lambek Calculus.

Examples: figure (6) shows that:

$$
s /(s / n p) s /((s / n p) \backslash s) \text { |- } s
$$

is a theorem of the Lambek Calculus.

Figure (7) below gives a correct net for the analysis of the sentence:

le livre dont je connais le titre est sur la table

\footnotetext{
${ }^{3} A$ is the usual designation of the Product-Free Lambek Calculus (see Zielonka 1981).
} 
(figure 7):

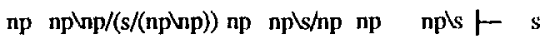

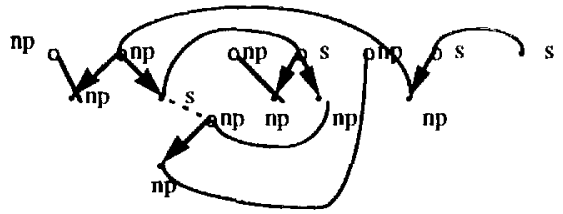

Theorem: (Categorization of Links) In a correct net, links are either identification links or connection links.

Corollary: A net is correct if and only if all nodes are either identified or connected.

Definition: we call Tree on words the tree obtained from a correct net by merging connected nodes and removing identification links. In the case of an identification link consisting in a type-1 edge, the link and the nodes linked by it are removed, and the adjacent type-2 edges are merged.

Example: figure (8): Pierre promet d Marie de venir

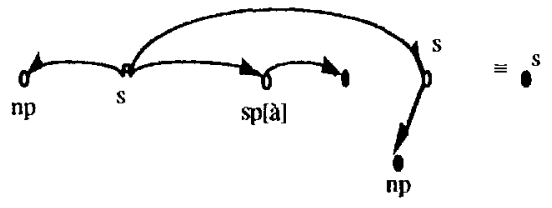

\section{Building Dependencies}

Obtaining a Dependency Structure from a tree on words amounts to doing little transformations on the correct net obtained by "equating" an ordered sequence of initial trees to a node representing a primitive category. These transformations involve indexing nodes in such a way that:

- indices of two different initial trees constitute two disjoint sets.

- indices inside an initial tree may be identical (if we want to express a coreference)

- linking two nodes results in identifying their indices.

Example: figure (9)

promet

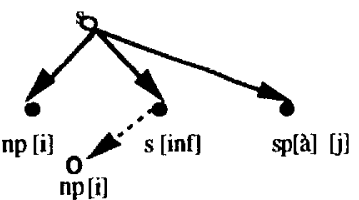

$a$

$\mathbf{s p}[\mathbf{a}][\mathbf{k}]$

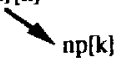

After getting a tree on words, we identify two distant nodes having the same index: we call the new node obtained: a shared node.

Finally, we can say that dependencies are obtained in the following stages:

1- indexing the nodes having the same label and belonging to different initial trees by different variables, taken in a set $(i, j, \ldots)$ (the distribution of indices inside an initial tree being set by the lexicon) [INDEXINGstep].

2- building the net corresponding to the assertion that the sequence is of type $s$ [NET-step].

3- suppressing the nodes identified by type-1 edges of the left-hand side and all the identification links [COLLAPSING-step].

4. if the same index appears on distinct nodes having the same label: merging them [MERGING-step] Example: figure (10)

Pierre promet à Marie de venir

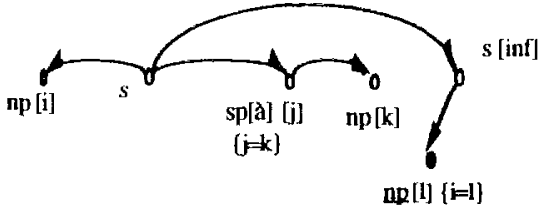

yields:

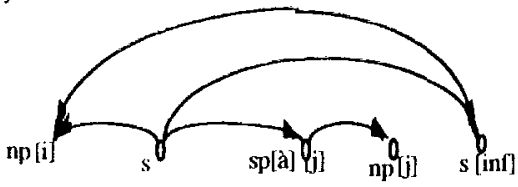

but, with permet instead of promet:

permet

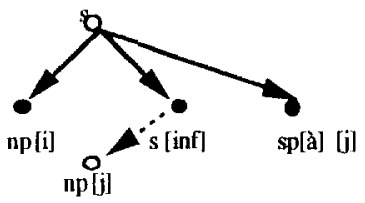

Pierre permet à Marie de venir:

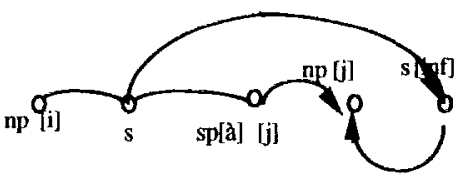




\section{Remarks and Conclusion}

This method of Connection Nets has many advantages over other methods.

Firstly, compared to classical strategies in the sequent calculus, it avoids spurious ambiguities and in so doing, it improves efficiency of searching the solution.

Secondly, compared to the method of Proof-Nets, it gives more clarity to the resulting structures. It is more efficient too, because the stage of checking the connexity when suppressing a branch of a type-1 link is replaced by a stage where the connexity is checked only once: when we have removed all the type-1 edges. The corresponding stage in Proof-Nets is usually named switching. In the early method by Girard which used the "long trip condition", there was a switch for each link and that gave an exponential-lime algorithm (in the number of links). In the method defined by Roorda, only type-1 links lead to switches. The reason lies in the necessity of checking that a type-1 link is not used to connect two subsets of the net, which would not be connected without it. (Let us recall that a type-1 link refers to a unary rule). In our method, switches are completely avoided.

Thirdly, it can be done incrementally. The reason is that the numbering of nodes is consistent with the order of initial trees. Thus, at each stage of the processing from left to right, we may have a beginning net which represents the present state of the processing. Here, the properties of left-associative grammars (Hausser 1990) are met.

Finally, a very few transformations are needed in order to obtain graphs on words which can be really interpreted as Dependency Structures.

\section{BIBLIOGRAPHY}

Ades, A. and Steedman, M.: 1982, 'On the Order of Words', Linguistics and Philosophy4, 517-558.

Barry, G. and Morrill, G.: 1990. (Eds) Studies in Calegorial Grammar. Edinburgh Working Papers in Cognitive Science, Volume 5. Centre for Cognitive Science, Edinburgh.

Barry, G \& Pickering, M.: 1990; 'Dependency and Constituency in Categorial Grammar', in Barry. G. and Morrill, G. 1990 and Lecomte, A. 1992.

Buszkowski 1986; 'Completeness Results for Lambek Syntactic Calculus', Zeitschr. f. math. Logik und Grundlagen d. Math. 32, 13-28.

Buszkowski 1988; 'Generative Power of Categorial Grammar',in R.Oehrle, E.Bach et D.Wheeler (eds) Categorial Grammars and Natural Languages Structures.

Danos, V. and Regnier, L.: 1989, The structure of multiplicatives' Arch. Math. Logic, 28, 181. 203.

Desclés, J.P.: 1990, Langages applicatifs, langues naturelles et cognition, Hermes, Paris.

Girard 1987; 'Linear Logic', Thoretical Computer Science 50, 1-102.
Hausser, R.: 1990, Computation of Language, Springer-Verlag, Berlin, Heidelberg.

Hendriks, H. and Roorda, D.: 1991, 'Spurious Ambiguity in Categorial Grammar', deliverable of the ESPRIT project BRA 3175 DYANA.

Hepple, M.: 1991, 'Efficient Incremental Processing with Categorial Grammar' Proceedings of $A C L$ 1991, Berkeley.

Hudson, R.A.: 1984, Word Grammar, Blackwell, Oxford.

Hudson, R. A.: 1990, English Word Grammar, Blackwell, Oxford.

Lambek, J.: 1958, 'The Mathematics of Sentence Structure',AmericanMath.Monthly 65,154-170.

Lambek, J.: 1961, 'On the Calculus of Syntactic Types' in Structure of Language and its Mathematical Aspects, AMS, Providence.

Lecomte, A.: 1990, 'Connection Grammars: a GraphOriented Interpretation of Categorial Grammars' in Lecomte, A. (ed), 1992.

Lecomte, A.: 1992. (ed.)Word Order in Categorial Grammar, ADOSA, Clermont-Ferrand.

Moortgat, M.:1988; Categorial Investigations. Logical and Linguistic Aspects of the Lambek Calculus, Dordrecht, Foris.

Moortgat, M.: 1990, 'Proof nets, partial deduction and resolution - Part 2' in Lecomte, A, 1992.

Oehrle, R.,Bach, E.and Whecler, D. (eds): 1988, Categorial Grammars and Natural Languages Structures, D. Reidel Publishing Company, Dordrecht et Boston.

Roorda, D.: 1990, 'Proof nets, partial deduction and resolution - Part 1 ' in Lecomte, A. 1992.

Roorda, D.: 1991, Resource Logics: Proof-theoretical Investigations, PhD Thesis, Faculteit van Wiskunde en Informatica, Amsterdam.

Stcedman, M.: 1991, Type-Raising and Directionality in Combinatory Grammar', Proceedings of ACL 1991, Berkeley.

Tesnière, L.: 1965, Eléments de syntaxe structurale, Klincksieck, Paris.

Wansing, H.: 1990, Formulaes-as-types for a Hierarchy of Sublagics of Intuitionistic. Propositional Logic, Gruppe für Logik, Wissentheorie und Information an der Freien Universităt Berlin.

Zielonka, W.: 1981, 'Axiomatizability of AjdukiewiczLambek Calculus by Means of Cancellation Schemes' Zeitschr. f. math. Logik und Grundlagen d. Math. 27, 215-224. 